\title{
LOS DESAFÍOS CONFRONTADOS POR LOS PROYECTOS CONVENCIONALES DE TRANSPORTE Y EL POTENCIAL DE LOS SISTEMAS INTELIGENTES DE TRANSPORTE PARA UNA CIUDAD EN DESARROLLO, LIMA, PERÚ
}

\section{CHALLENGES TO CONVENTIONAL TRANSPORTATION PROJECTS AND THE POTENTIAL FOR INTELLIGENT TRANSPORTATION SYSTEMS IN A DEVELOPING CITY, LIMA, PERU}

\section{RESUMEN}

\author{
Manuel J. Martínez
}

Este artículo examina los desafíos que encuentran los proyectos convencionales de transporte urbano cuando se implementan durante el proceso de motorización de Lima. Como solución potencial, se recomienda que se priorice la investigación y desarrollo de los Sistemas Inteligentes de Transporte para Lima.

Palabras clave: Sistemas Inteligentes de Transporte, Telecomunicaciones, Transporte Urbano, Política de Transporte Urbano.

\begin{abstract}
This paper presents the challenges to the implementation of conventional transportation projects in developing cities with motorization process and recommends research in Intelligent Transportation Systems.
\end{abstract}

Key words: Intelligent Transportation Systems, Telecommunications, Urban Transportation, Urban Transportation Policy

\section{A. LAS CICLOVÍAS}

Las ciclovías son recomendables para ciudades en desarrollo por ser económicas, eficientes, e indicadas para los sectores de bajos ingresos [1]

En Lima, el Plan de Desarrollo Metropolitano de 1991 incluyó siete ciclovías además de la Ciclovía de Av. Arequipa [2]. En 1995 ya estaban operativas tres ciclovías, mientras que el World Bank (WB) financiaría dos ciclovías adicionales con \$2M [3]. Se esperaba que las nuevas ciclovías incrementarían la demanda de bicicletas de $2 \%$ a $10 \%$ del total de viajes [4]. Además, desde 2002, el Global Environmental Facility (GEF) dedicó $\$ 4 \mathrm{M}$ a ciclovías de Lima bajo la supervisión del WB [5]

En el 2002, el Fondo Nacional de Ambiente (FONAM) estudió la demanda de las ciclovías y encontró que el riesgo de accidentes era el freno principal de la demanda, pero que también había un problema de imagen puesto que las bicicletas se podían ver como deporte ó como señal de pobreza [6]. El más reciente Plan Ciclovial presenta unos flujos muy reducidos de 60 y 90 bphpd en hora pico, es decir, apenas el $3.5 \%$ del uso de la infraestructura, aunque el Plan sigue expresando altas expectativas por el buen clima de Lima, el porcentaje de población de menos de 30 años, y el alto porcentaje del sector de pocos ingresos [7]

En conclusión, las altas expectativas de las ciclovías confrontan el desafío de la realidad de una demanda muy reducida. Todavía no se ha estudiado el hecho de que casi todo el mercado potencial de usuarios de ciclovías lo constituyen usuarios de un transporte público de amplia cobertura y de pasaje muy económico

\section{B. EL TRANSPORTE DE BUSES RÁPIDOS}

El transporte de buses rápidos TBR es un nuevo modo de transporte, originado en Latinoamérica, el cual se puede implementar por etapas a partir del corredor exclusivo de buses, incrementando características adicionales de alta capacidad, hasta el punto en que se comienzan a parecer a trenes urbanos. Usualmente, se recomienda hacer una evaluación de alternativas TBR versus trenes, que dé mayor información a lasinstituciones que toman las decisiones correspondientes. Es en este contexto que el primer estudio para elevar la calidad del corredor exclusivo de buses se realiza en 1998, el mismo año en el cual se realiza el estudio del proyecto de trenes urbanos escogiendo ambos la ruta de la Vía Expresa como acceso Sur-Norte al Centro de Lima [8]. El estudio de corredor exclusivo de buses, financiado por el WB, consistía en una red de $67 \mathrm{~km}$. de corredores de buses incluyendo a los $8 \mathrm{~km}$. de la Vía Expresa [8]. En el 2001, una evaluación que incluyó trenes, encontró que la mejor alternativa era la de tren por la Vía Expresa insertado en los $67 \mathrm{~km}$. de corredores exclusivos de buses [9]. La decisión final sobre la Vía Expresa se realizó en 2004 cuando fue oficialmente asignada a Protransporte para construir el TBR [10]. Esta disputa por la Vía Expresa refleja la escasez de infraestructura de transporte que tiene Lima.

El corredor exclusivo de buses, llamado Vitrina, comprendía un corredor de $18.4 \mathrm{~km}$. a un costo de $\$ 21.5 \mathrm{M}$. La ruta incluía la Vía Expresa y el Cono Nor-Este de Lima. El sistema disponía de las siguientes características de alta capacidad: paradero separado de la vía, pre-pago del pasaje, buses estándares de dos puertas y carriles especiales de sobre-paso ([11]. En el 2001, estudios adicionales de ingeniería de mayor detalle,

\footnotetext{
${ }^{a}$ Doctor of Philosophy in Transportation, Major in Transportation Engineering, del New Jersey Institute of Technology, y también Humphrey
} Fellow especializado en Urban Transportation Planning and Policy, de la Rutgers, The State University of New Jersey, y Economista Colegiado de la Pontificia Universidad Católica del Perú, Registro CEL 4047 
establecieron que los costos del corredor Vitrina se ampliarían a $\$ 50 \mathrm{M}$, de los cuales, $\$ 28 \mathrm{M}$ se dedicarían a elevar la calidad de los paraderos-estaciones del sistema [9]

En el 2002, el WB incrementó su porción de financiamiento a $\$ 120 \mathrm{M}$ con el objetivo de elevar la calidad del proyecto hasta la de un TBR, comprendiendo una ruta de $40 \mathrm{~km}$. porque alcanzaría dos conos: Cono Norte y Cono Nor-Este [5]. Luego, se decidió que el nuevo TBR incluyera una extensión al Cono Sur por Chorrillos con las siguientes características de alta capacidad: estaciones de plataforma elevada tipo tren, carriles de sobrepaso en las estaciones, y buses articulados [12]. En el 2003, la aproximación por la Vía Expresa al Centro de Lima fue diseñado en superficie, aunque se recomendó un estudio especial para la intersección entre la Vía Expresa y la Plaza Grau para sugerir soluciones al tráfico intenso de esta intersección [13]. La misma serie de estudios recomendó que la ruta se dedicara solamente al Cono Norte y no al Cono Nor-Este, recortando el kilometraje de la ruta [13]. Finalmente, en el 2004, en el estudio de la intersección de Vía Expresa con la Plaza Grau se recomendó la aproximación en túnel para resolver el problema del tráfico intenso de esta intersección, y además construir una estación central subterránea más allá de la intersección. Así el costo del proyecto TBR se incrementó a un total de $\$ 206 \mathrm{M}$, de los cuales el WB y el Banco Interamericano de Desarrollo (BID) aportaban $\$ 90 \mathrm{M}$ y la Municipalidad Metropolitana de Lima (MML) una contrapartida de \$44M [14]

En consecuencia, el proyecto de TBR ha venido incrementando su costo desde un corredor exclusivo de buses de $18.4 \mathrm{~km}$. a $\$ 21.5 \mathrm{M}$ hasta un TBR de $33 \mathrm{~km}$. a $\$ 206 \mathrm{M}$, casi cinco veces el costo por kilómetro original, mayormente incrementos ligados a obras civiles, otra señal de la escasez de infraestructura de transporte. Aunque es notorio que no se incluye un puente sobre el Río Rímac, uno de los principales cuellos de botella de la dirección Norte-Sur.

Los costos sociales presupuestados también se incrementaron. En el proyecto original se diagnosticó que todos los operadores de las rutas superpuestas ingresaban al corredor Vitrina, con la excepción de buses con más de 20 años de uso, y algunos combis, hasta un total de 1,583 vehículos [11].

En el 2002, cuando el corredor Vitrina se eleva de calidad a un proyecto TBR, Protransporte, que además de administrar el proyecto también era el órgano planificador de transporte de Lima, diagnosticó un 50\% de sobreoferta del transporte público con respecto a la situación de 1990 y recomendó el ordenamiento total del sistema del transporte público [12]. Traducido a una meta de política, esta cifra significaría la erradicación de 30,500 vehículos del transporte público.

Paralelamente, en el 2002, los costos sociales del TBR fueron calculados en 10,481 vehículos afectados, una cifra siete veces mayor que el corredor Vitrina, compuesto por los operadores actuales a ser erradicados de la infraestructura de transporte existente, la que pasaría a ser usada por el TBR en exclusividad, incluyendo el Puente del Ejército sobre el río Rímac [12, 15].Para financiar los costos sociales de erradicar los 10,481 vehículos afectados por el TBR, el Global Environmental Facility (GEF) proporcionaría una ayuda de unos \$10M con el fin de comprar cada combi a $\$ 3,000$ y cada bus de más de 20 años de uso a $\$ 7,000$ [15]. Sin embargo, se trata de un fondo que financiaría la erradicación de sólo unos 3,500 vehículos en primera instancia. Además, el precio de compra de $\$ 3,000$ por combi no parece ser voluntario, puesto que un combi puede recuperar esta suma como ganancia neta en 150 días de trabajo [16]

En resumen, los costos sociales presupuestados han crecido a medida que los estudios se han hecho más precisos y que se ha levantado la calidad del proyecto a un proyecto TBR. Estos costos sociales no están financiados, puesto que cuenta con solo $\$ 10 \mathrm{M}$. Si se consideraran los 30,000 vehículos de sobreoferta se necesitarían $\$ 90 \mathrm{M}$, y si se considerara como precio el valor de ingreso esperado el esta suma se incrementaría considerablemente.

En conclusión, estos resultados expresan los desafíos confrontados por el TBR en el contexto de una motorización que incluye a vehículos de transporte público y que ocurre sobre una escasa infraestructura de transporte: escalamiento de costos económicos y sociales

\section{EL PROCESO DE MOTORIZACIÓN HASTA LA ACTUALIDAD}

El proceso de motorización en el Perú comprende no solamente el incremento explosivo del transporte privado de autos por la masiva compra de automóviles, sino también el incremento masivo de transporte público.En 1992, los vehículos de transporte público había llegado a 10,438, pero aún se necesitaban más unidades [2]. En ese momento los combis eran parte de la solución a la escasez de vehículos de transporte urbano, por lo cual se les dio un impulso en la forma de la des-regulación del transporte público que declaraba libertad de entrada al mercado y de precios [2].

En Lima, en una encuesta de 1998, resultó que el 32\% de los usuarios de transporte público prefería combis, mientras que sólo $23 \%$ prefería los buses estándar, principalmente porque los combis eran veloces, siendo la velocidad la característica más importante para el $40 \%$ de los usuarios [17]. Pronto, los combis se convirtieron en vehículos de transporte público masivo, en 1997 los combis servían el $49 \%$ de los viajes en transporte público de Lima Metropolitana, mientras que en el 2005 servían el $44 \%[18,19]$

Además, el incremento de vehículos de transporte público era una solución al problema de la creación de empleo para los pobres sin necesidad de subsidios, puesto que era una solución típica de $s$ libre mercado en ciudades en desarrollo [20]

Durante el período en que se des-reguló el transporte urbano, el número de vehículos creció desde 10,500 en 1990 a 47,000 en 1997, a una tasa anual de crecimiento de $24 \%$, y luego a 61,000 vehículos en el 2004 a una tasa anual de crecimiento de $4 \%$ [21, 22]. Asimismo, la cantidad de autos particulares creció de 266,000 en 1989 a 322,000 en 1994, a una tasa anual de crecimiento de 
$4 \%$, y luego a 421,000 autos en 2004 , a una tasa anual de crecimiento de $3 \%[3,19]$

Este proceso de motorización, que incluye al transporte público en el caso de Lima, al llegar antes que los proyectos convencionales; llámense ciclovías y TBR, les impone a estos proyectos los desafíos descritos en los puntos anteriores.

\section{EL POTENCIAL DE LOS SISTEMAS INTELIGENTES DE TRANSPORTE}

Los Sistemas Inteligentes de Transporte (SIT) consisten en la aplicación de telecomunicaciones, informática y electrónica al campo del transporte. Entre los beneficios medidos por ciudades que ya han desplegado servicios SIT se encuentran los siguientes: i) reducción de la congestión producida por incidentes (accidentes, marchas, espectáculos, etc.), ii) reducción de la contaminación y del consumo de combustible al reducir las emisiones por kilómetro, e iii) incremento de la productividad del sistema de transporte al reducir el tiempo de viaje [23]

Actualmente, ciudades en desarrollo están implementando sistemas SIT porque han mejorado las condiciones para la introducción de estos sistemas: al adecuar tecnologías ya comprobadas en los países en desarrollo y al disponer de una infraestructura difundida de teléfonos celulares y de Internet [24]. En Lima, se ha pronosticado que el proceso de motorización se acentuará en los próximos años, creciendo el porcentaje de transporte en autos de $15 \%$ a $22 \%$ en 2025 , mientras que el total de viajes motorizados crecerá en $50 \%$. El proceso de motorización será rápido debido al futuro progreso económico. Este proceso será de tal magnitud, que incluso si se implementaran todos los proyectos de trenes urbanos, TBRs, y corredores exclusivos de buses, todavía la congestión crecerá de $8 \%$ a $15 \%$ de las vías de transporte, y la velocidad bajará de 17 a 12km-h [19]. En consecuencia, habrá un margen considerable para aplicar nuevas tecnologías para mejorar la eficiencia del transporte urbano de Lima y obtener beneficios sociales de reducir la congestión y la contaminación. Es de conocimiento que las infraestructuras de teléfonos celulares e Internet se están extendiendo continuamente en Lima y a precios cada vez más económicos, haciendo de telecomunicaciones un recurso de uso intenso. Sin embargo, se necesita que se apoye la investigación científica y tecnológica de estos SIT con el fin de adecuar estos servicios a la realidad de Lima en forma apropiada, con costo-efectividad y sin costos sociales

\section{E. CONCLUSIONES Y RECOMENDACIONES}

- Los proyectos convencionales de transporte, confrontan los desafíos de falta de demanda en el caso de las ciclovías, y escalamiento de costos económicos y sociales en el caso del TBR.

- Los desafíos surgen de los rasgos especiales del proceso de motorización en Lima: i) escasa infraestructura de transporte y ii) gran incremento de los vehículos de transporte público que copan la escasa infraestructura. Este proceso de motorización llegó antes que estos proyectos convencionales se implementaran

- En el futuro, el proceso de motorización se acentuará incrementando la congestión y contaminación en Lima

- Una nueva tecnología que todavía no se ha implementado en Lima son los servicios SIT (Sistemas Inteligentes de Transporte), los cuales podrían ser una solución potencial puesto que ha obtenido buenos resultados en países desarrollados, donde prevalece el uso intensivo de los automóviles particulares en situación de congestión, pero que necesita adecuarse a Lima. Además, la extensión de la infraestructura de telecomunicaciones se ha extendido de tal manera de hacer propicio el desarrollo de los servicios SIT.

- Se recomienda la investigación y desarrollo de nuevos servicios SIT adecuados a la realidad de Lima, y que provean de soluciones costo-efectivas, y sin costos sociales

\section{AGRADECIMIENTOS}

EI CONCYTEC, Consejo Nacional de Ciencia y Tecnología financia el Contrato 194-2005-CONCYTECOAJ dentro del cual se inscribe el presente artículo (marzo 2006).

\section{REFERENCIAS}

[1] World Bank. 1975. Urban Transport Sector Policy Paper. Washington, DC

[2] Municipalidad Metropolitana de Lima. 1992. Plan de Desarrollo Metropolitano de Lima-Callao 1990-2010 Primer Volumen. Lima, Perú.

[3] Iturregui, Patricia. 1996. Problemas Ambientales de Lima. Algunas Propuestas y la Necesidad de una Agenda 21 Local. Fundacion Alemana Friedrich Ebert. Lima

[4] Peters, Deike. 1997. "Bikeways Come to Lima's Mean Streets". IN Sustainable Transport. Winter 1997. pp:1617. ITDP. New York

[5] Bonini, Astra, et al. 2002. "Green Fund Switches to Sustainable Transportation". IN Sustainable Transport. 14 pp:24-25. ITDP. NY

[6] FONAM. 2002. Conocimiento, Actitudes y Practicas (CAP) sobre la Bicicleta. Analisis Integral General. Entrevistas en Profundidad. Lima, Peru

[7] CIDATT, Taryet SRL, Fundacion Ciudad Humana. 2005. Plan Maestro de Ciclovias para Lima y Callao. Primer Borrador del Informe Final. FONAM. Lima, Peru

[8] Perera Diaz, Fernando. 1998. "Propuesta de Reordenamiento del Transporte en Lima y Callao" IN Transporte Urbano y Ambiente. Bases para una Política Ambiental en el Transporte Urbano pp:183-188. Edited by CONAM. Lima, Peru 
[9] (9) Consejo de Transporte de Lima y Callao. 2001. Plan Estratégico de Transporte Urbano. Proyectos Metropolitanos. Informe Final. Lima, Perú

[10] Municipalidad Metropolitana de Lima. 2004. Ordenanza 682 Declara Intangibilidad y Reserva de las Áreas Destinadas al Programa de Transporte Urbano de Lima. Lima, Perú

[11] Consejo de Transporte de Lima y Callao. 1999. Vitrina, Asistencia Técnica en Estudios de Transporte Urbano para el Área Metropolitana de Lima y Callao. Informe Final. Informe de Resultados. Preparado por Booz-Allen y Hamilton / Barriga-D'Allorto / Wilbur Smith. Lima, Perú

[12] Protransporte. 2002. Estudio de los Impactos Ambientales en el Area de Influencia Directa del Corredor Segregado de Alta Capacidad/PTUL. Corredores Segregados de Alta Capacidad. Prepared by GETINSA and TARYET. Financed by the InterAmerican Development Bank. Lima, Peru

[13] Protransporte. 2003. Memoria y Síntesis de los Estudios Tecnicos y Ambientales del Corredor Segregado de Alta Capacidad (COSAC I) y sus Terminales de Transferencia (COSAC 1). Prepared by Getinsa and Taryet. Lima

[14] Protransporte. 2004. Presentación Dirección Ejecutiva. Noviembre 2004. Lima

[15] FONAM. 2002. Informe Final Diseño de Incentivos y Plan para el Desguace de los Vehículos Obsoletos del Transporte Público de Pasajeros. Preparado por Swiss Contact Services Peru y Recursos SAC Consultoría Empresarial. Lima, Perú

[16] Swiss Contact. 2003. Informe Final Focus Groups Aceptación y Expectativas de un Sistema de Incentivos para el Desguace de Vehículos Obsoletos de Transporte Público de Pasajero. Lima, Perú

[17] Programa Urbano DESCO. 2002. Estudio y Valoración de los Beneficiarios proyecto Transporte Público Urbano Lima/Beneficiary Assessment (BA). Elaborado para el Banco Mundial y Protransporte. Lima, Perú

[18] INEI. 1998. Perú: Perfil del Transporte Urbano en los Hogares-ENAHO 1997

[19] Ministerio de Transportes y Comunicaciones. 2005. The Master Plan for Lima and Callao Metropolitan Area Urban Transportation in the Republic of Peru (Phase 1) Final Report (Volume 1) Part 1 Current Conditions. Prepared by Yachiyo Engineering Co., Ltd. and Pacific Consultants International. Financed by JICA

[20] De Soto, Hernando. 2000. El Misterio del Capital. Editora El Comercio SA. Lima

[21] Consejo de Transporte de Lima y Callao. 1998. Asistencia Técnica para la Evaluación y Consulta a Operadores del Transporte Público del Área Metropolitana de Lima y Callao. Informe de Resultados. Lima, Perú

[22] Municipalidad Metropolitana de Lima. 2004. Estadísticas. Lima, Perú

[23] ITS America. 1994. Assessment of the Benefits and Costs of the ITS User Benefits. Benefits, Evaluation and Costs Committee. Workshop Proceedings Newport Beach, California

[24] Yokota, Toshiyuki, Haruo Ishida, and Kotaro Kato. 2004. "Deployment of Intelligent Transportation Systems in Countries with Developing and Transitional Economies" IN Journal of the Transportation Research Board 1886. pp:1-9. Washington, DC

Email: mjmartinez@pucp.edu.pe avancetransporteperuano@yahoo.com 